Nani pul abi I i ty Neasur es taki ng Necessary Joi nt Tor ques for $G$ asping i nto consi der at i on

\begin{tabular}{|l|l|}
\hline 著者 & Wat anabe Tet suy ou \\
\hline $\begin{array}{l}\text { j ournal or } \\
\text { publ i cat i on t i t I e }\end{array}$ & $\begin{array}{l}\text { Proceedi ngs of the I EEE/RSJ I nt er nat i onal } \\
\text { Conf er ence on I nt el I i gent Robot s and Syst ens } \\
\text { ( I ROS) }\end{array}$ \\
\hline vol une & 2010 \\
\hline number & art. no. 5651352 \\
\hline page r ange & $598-603$ \\
\hline year & $2010-01-01$ \\
\hline URL & ht t p: //hdl . handl e. net /2297/35245 \\
\hline
\end{tabular}




\title{
Manipulability Measures taking Necessary Joint Torques for Grasping into consideration
}

\author{
Tetsuyou Watanabe
}

\begin{abstract}
This paper presents new manipulability measures to evaluate how much easily the robot manipulates the grasped object, simultaneously taking how much magnitude of joint torque we need to keep grasping into consideration. For the purpose, we use operation range. The operation range is for actuator attached to every joint of robot and provides generable joint torque and velocity and their relation (between generating torque/velocity and addable velocity/torque). While we introduced a manipulability measure using the operation range in our previous paper, it was for a limited class due to large computational effort and we could not evaluate whole space of object velocity and could not consider whole space of external wrench. This paper proposes new manipulability measures which can evaluate whole space of object velocity, taking the effect of external wrench in whole space into consideration.
\end{abstract}

\section{INTRODUCTION}

Manipulability is a well known concept to evaluate the performance of robotic manipulator [1]. For a single-arm manipulator, it is defined as the set of generable endeffector velocity in the task space when the set of generable joint velocity is given. When the given set of joint velocity is a unit ball, the set of endeffector velocity becomes an ellipsoid. The ellipsoid is called manipulability ellipsoid. The volume of the ellipsoid can be regarded as a quality measure to evaluate the performance in velocity domain. It is called manipulability measure. Based on the manipulability, many quality measures such as condition number are proposed [1].

This concept can be extended to the general constraining system such as a robotic hand [2]-[6]. In a general constraining system, object velocity is evaluated instead of endeffector velocity. For a dual-arm system, Chiacchio et al. [2] discussed manipulability. Bicchi et al. [3] analyzed manipulability for general grasping system including whole arm manipulation system. After that, Bicchi et al. [4], Wen et al. [5], and Park et al. [6] analyzed manipulability for general constraining systems with underactuated joints.

In a grasping system, grasping itself is a key issue. Therefore, if evaluating manipulability, we need to take whether being able to keep grasping or not into consideration simultaneously. However, the above researches did not take it into consideration by assuming force closure grasp (force closure is defined that any force and moment in any direction can be applied to the object). As a consequence, we could not take the effects of friction, object gravity, contact state, and so on into consideration. For example, the evaluation

This work was partially supported by KAKENHI(20760162)

T. Watanabe is with College of Science and Engineering, Kanazawa University, Kanazawa, 920-1192, Japan te-watanabe@ieee.org when grasping a light object is the same as the evaluation when grasping a heavy object. It can be said that the above analyses were not for a grasping system but for closed-chain manipulators/robots.

To cope with the issue, we presented a method to analyze a manipulability in the previous paper [7]. However, the obtained results were limited. In this paper, we present new manipulability measures for grasping system. Here are the main contributions of this paper (especially comparing with the previous paper [7]).

1) Required external wrench set taking external wrench in any direction into consideration: In order to take the effect of external wrench exerted on the object (for example, gravitational force) into consideration, we derive required joint torque to balance the external wrench and keep stable grasping. For the purpose, we introduce required external wrench set (REWS) [8], [9], which is defined as a set of external wrench required to be balanced. We suppose that the grasp is stable if any arbitrary external wrench contained in REWS can be balanced. In the previous paper, we used REWS expressed as a convex polyhedron. However, there are some cases when REWS cannot be expressed by a convex polyhedron. Suppose the case, for example, when external wrench in any arbitrary direction can be exerted on the object. REWS should be expressed by an ellipsoid or hypersphere. We can approximate the set by a convex polyhedron, but it is difficult in a high dimensional space, since a large number of vertices or faces are needed. This paper presents a way to deal with REWS expressed by not only a convex polyhedron but also an ellipsoid, and to derive minimum required joint torques for the stable grasping.

2) Not-convex operation range: Based on the minimum required joint torques for stable grasping, we derive maximum usable joint velocities. For the purpose, we use an operation range (shown in Fig.1) of actuator attached to every joint of robot (operation range is originally used for motor selection). The operation range provides not only information about how magnitudes of torque and velocity the actuator can stably generate, but also the relationship between generating velocity/torque and addable torque/velocity. The rigid line in Fig.1 shows the operation range derived by supposing to control the motor with nominal voltage. There are some cases when we can get only the information about power, maximum generable velocity and torques. In such a case, we will derive the operation range by using the relationship; power $=$ torque $\times$ velocity (see the area surrounded by the dot line in Fig.1). The derived operation range is not always convex. The previous paper could deal with only convex 


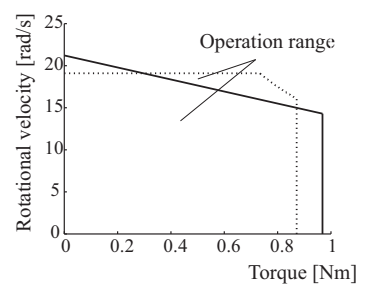

Fig. 1. Operation range of torque and velocity (maxon DC motor Amax32 (20W) with gear (ratio: 23:1));Area of rigid line: supposing to control with nominal voltage, Area of dot line: supposing the case when power and maximum torque and velocity are only given and $70 \%$ of the power and $90 \%$ of the maximum torque and velocity are used.

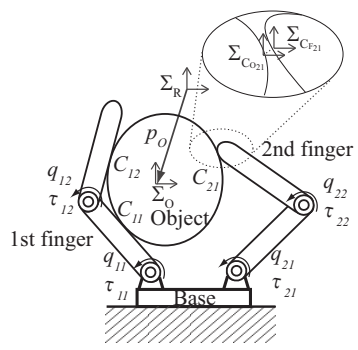

Fig. 2. Target System

operation range. In this paper, we present a way to deal with a not-convex operation range.

3) Manipulability measure to evaluate the whole space of object velocity: Using the derived maximum usable joint velocity, we derive the generable object velocity. The previous paper evaluated a limited class of object velocity due to the high computational complexity. We needed to deal with a lot of variables in a quite high dimensional space. Therefore, its application fields were very limited. For example, it is hard to apply the previous approach to motion planning, especially on line. This paper presents manipulability measures which can be more easily derived and can evaluate the whole space of the object velocity. We focus on the magnitude of the object velocity. We derive the maximum object velocity available in any direction, and the maximum generable object velocity and its direction. The former one corresponds to the radius of the hypersphere inscribed in the set of the generable object velocity while the latter one corresponds to the radius of the hypersphere circumscribed in the set of the generable object velocity.

\section{Problem Definition}

\section{A. Target system}

The target system is shown in Fig.2. In this paper, we consider a general grasping system where an arbitrary shaped rigid object is grasped by $N$ fingers of a robotic hand. The nomenclatures are listed at appendix. We define that the contact state is any of the following two states: 1) F-point : the contact point with friction, 2) N-point : the contact point without friction. Translational force in any direction can be applied to the object at F-point under the frictional condition while only normal force can be applied to the object at $\mathrm{N}$ point.
We note that every contact position, every contact state, and every frictional coefficient are all given. Note also that the absolute magnitude of minimum generable joint torque/velocity is equal to the absolute magnitude of maximum generable joint torque/velocity.

\section{B. Problem Definition}

Here, we give several definitions and define the problem handled in this paper.

Joint Torque-velocity Pair Set (TVS): The set of generable joint torque and velocity at each joint, given by the corresponding actuator and gear specifications, is named joint torque-velocity pair set (TVS).

The specification for actuators (operation range) is usually given with respect to the absolute values of torque and velocity. We express generable maximum absolute values of joint torque and velocity with $\left|\tau_{i j_{\max }}\right|$ and $\left|\dot{q}_{i j_{\max }}\right|$. Let $\left|\dot{q}_{i j_{U \max }}\right|(\geq 0)$ be the usable maximum absolute value of joint velocity, determined by currently generating joint torque. Similarly, let $\left|\tau_{i j_{U \max }}\right|(\geq 0)$ be the usable maximum absolute value of joint torque, determined by currently generating joint velocity. We describe this relationship with the following functions.

$$
\begin{aligned}
\left|\dot{q}_{i j_{U \text { max }}}\right| & =\boldsymbol{\xi}_{i j}^{\tau \rightarrow \dot{q}}\left(\left|\tau_{i j}\right|\right), \\
\left|\tau_{i j_{U \text { max }}}\right| & =\boldsymbol{\xi}_{i j}^{\dot{q} \rightarrow \tau}\left(\left|\dot{q}_{i j}\right|\right)=\left(\boldsymbol{\xi}_{i j}^{\tau \rightarrow \dot{q}}\right)^{-1}\left(\left|\dot{q}_{i j}\right|\right) .
\end{aligned}
$$

This function $\boldsymbol{\xi}_{i j}^{\tau \rightarrow \dot{q}}$ can be derived from the actuator and gear specifications. For example, if using DC motor under the constant nominal voltage, $\boldsymbol{\xi}_{i j}^{\tau \rightarrow \dot{q}}$ can be derived by utilizing maximum speed under the voltage, maximum torque, and torque-speed constant. An example of TVS is the area surrounded by the rigid lines in Fig.1, and $\boldsymbol{\xi}_{i j}^{\tau \rightarrow \dot{q}}$ gives the rigid line. In this case, the TVS is convex. If we can not get the information about torque-speed constant, we use power. Let $\psi_{i j}$ be the constant power for the evaluation. Then, $\boldsymbol{\xi}_{i j}^{\tau \rightarrow \dot{q}}$ can be expressed by

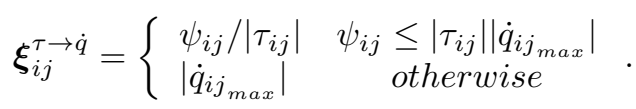

An example of TVS derived by this way is the area surrounded by the dot lines in Fig.1.

External wrench such as gravitational force is exerted on the object. In order to preserve stable grasping, we need to balance such kinds of external wrenches. To consider the effect of such kinds of external wrenches, we define required external wrench set:

Required External Wrench Set (REWS): The set of object's external wrench required to be balanced is named required external wrench set (REWS). We suppose that the grasp is stable if any arbitrary external wrench contained in REWS can be balanced.

REWS is assumed to be given by convex polyhedron or ellipsoid;

$$
\begin{aligned}
& \mathcal{S}_{\text {rew }}^{\text {pol }}=\left\{\boldsymbol{w}_{\text {ex }} \mid \boldsymbol{w}_{\text {ex }}=\sum_{i=1}^{n_{\text {rew }}} \lambda_{i} \boldsymbol{w}_{v_{i}}, \sum_{i=1}^{n_{\text {rew }}} \lambda_{i}=1, \lambda_{i} \geq 0\right\}, \\
& \mathcal{S}_{\text {rew }}^{\text {elip }}=\left\{\boldsymbol{w}_{\text {ex }} \mid \boldsymbol{w}_{\text {ex }}^{T} \boldsymbol{M}_{w}^{T} \boldsymbol{M}_{w} \boldsymbol{w}_{\text {ex }} \leq 1\right\}
\end{aligned}
$$


Here, $\boldsymbol{w}_{e x}$ denotes external wrench, $\boldsymbol{w}_{v_{i}}$ denotes $i$ th vertex of the convex polyhedron, $n_{\text {rew }}$ denotes the number of the vertices, $\boldsymbol{M}_{w}$ denotes a weight matrix, for example, to take the difference between the units of force and moment into consideration, or to normalize the magnitude of the external wrench. $\lambda_{i}$ is a parameter to express the convex polyhedron. Criterion: In this paper, we consider the following criteria: $\alpha_{\max }^{\text {all }}$ : the maximum magnitude of the object velocity available in any arbitrary direction; $\alpha_{\max }$ : the maximum magnitude of the generable object velocity.

$\alpha_{\max }^{\text {all }}$ expresses object velocity usable in any direction. It is useful, for example, when designing controller or when we do not know which direction of object velocity we will need. We can regard it as the distance from a kind of singular configuration where object velocity in a certain direction is not available. On the other hand, $\alpha_{\max }$ is to evaluate which direction we can generate maximum object velocity and its magnitude. $\alpha_{\max }^{a l l} / \alpha_{\max }$ can be used to evaluate how uniformly we can generate the object velocity.

Then, we define the following problem:

Problem : Suppose that TVS and REWS are given. In this case, find $\alpha_{\max }^{a l l}$ and $\alpha_{\max }$ under the condition that any external wrench contained in REWS can be balanced.

\section{Manipulability MEASURES BASED ON TVS}

First, we consider kinematics, statics, and frictional constraints. Then, we derive new manipulability measures.

\section{A. Kinematics}

With respect to contact point $C_{i j}$, the relationship between the velocities of $\boldsymbol{p}_{C_{F_{i j}}}$ and $\boldsymbol{q}_{i}$, and the relationship between the velocities of $\boldsymbol{p}_{C_{O_{i j}}}$ and $\boldsymbol{r}$ are given by

$$
\dot{\boldsymbol{p}}_{C_{F_{i j}}}=\boldsymbol{J}_{i j} \dot{\boldsymbol{q}}_{i}, \quad \dot{\boldsymbol{p}}_{C_{O_{i j}}}=\boldsymbol{G}_{i j}^{T} \dot{\boldsymbol{r}}
$$

where $\boldsymbol{J}_{i j} \in \mathcal{R}^{d \times M_{i}}$ denotes Jacobian matrix and $\boldsymbol{G}_{i j}^{T}=$ $\left[\begin{array}{ll}\boldsymbol{I} & -\left[\left(\boldsymbol{p}_{C_{O_{i j}}}-\boldsymbol{p}_{o}\right) \times\right]\end{array}\right] \in \mathcal{R}^{d \times D}$. Here, $\boldsymbol{I}$ represents an identity matrix, $[\boldsymbol{a} \times]$ represents a skew symmetric matrix equivalent to the cross product operation $([\boldsymbol{a} \times] \boldsymbol{b}=\boldsymbol{a} \times \boldsymbol{b})$.

The relationship between $\dot{\boldsymbol{p}}_{C_{F_{i j}}}$ and $\dot{\boldsymbol{p}}_{C_{O_{i j}}}$ is written by

$$
\boldsymbol{H}_{i j}\left(\dot{\boldsymbol{p}}_{C_{F_{i j}}}-\dot{\boldsymbol{p}}_{C_{O_{i j}}}\right)=\boldsymbol{o}, \boldsymbol{H}_{i j}=\left\{\begin{array}{cl}
\boldsymbol{I} & \text { for F-point } \\
\boldsymbol{n}_{i j}^{T} & \text { for N-point }
\end{array},\right.
$$

where $\boldsymbol{o}$ denotes a zero vector.

Then, using the following matrices: $\boldsymbol{J}_{i} x=\operatorname{col}\left[\boldsymbol{J}_{i j}\right] \in$ $\mathcal{R}^{L_{i} d \times M_{i}}, \quad \boldsymbol{H}_{i}=\operatorname{diag}\left[\boldsymbol{H}_{i j}\right] \in \mathcal{R}^{L_{c i} \times L_{i} d}, \quad \boldsymbol{G}_{i}=$ $\left[\begin{array}{llll}\boldsymbol{G}_{i 1} & \boldsymbol{G}_{i 2} & \cdots & \boldsymbol{G}_{i L_{i}}\end{array}\right] \in \mathcal{R}^{D \times L_{i} d}, \boldsymbol{J}_{H i}=\boldsymbol{H}_{i} \boldsymbol{J}_{i} \in$ $\mathcal{R}^{L_{c i} \times M_{i}}, \boldsymbol{G}_{H i}=\boldsymbol{G}_{i} \boldsymbol{H}_{i}^{T} \in \mathcal{R}^{D \times L_{c i}}$, we aggregate the relationships with respect to every finger and get the following contact velocity:

$$
\dot{\boldsymbol{p}}_{C i}=\boldsymbol{J}_{H i} \dot{\boldsymbol{q}}_{i}=\boldsymbol{G}_{H i}^{T} \dot{\boldsymbol{r}} .
$$

Using the following matrices: $\dot{\boldsymbol{p}}_{C}=\operatorname{col}\left[\dot{\boldsymbol{p}}_{C i}\right] \in$ $\mathcal{R}^{L_{c}}, \quad \boldsymbol{J}_{H}=\operatorname{diag}\left[\boldsymbol{J}_{H i}\right] \in \mathcal{R}^{L_{c} \times M}, \quad \boldsymbol{G}_{H}=$ $\left[\begin{array}{llll}\boldsymbol{G}_{H 1} & \boldsymbol{G}_{H 2} & \cdots & \boldsymbol{G}_{H N}\end{array}\right] \in \mathcal{R}^{D \times L_{c}}$, we aggregate the relationships for all fingers and obtain

$$
\dot{\boldsymbol{p}}_{C}=\boldsymbol{J}_{H} \dot{\boldsymbol{q}}=\boldsymbol{G}_{H}^{T} \dot{\boldsymbol{r}} .
$$

\section{B. Statics and frictional constraints}

From (8) and the principle of virtual work, considering gravitational term of robot, the following relation is obtained

$$
\left[\begin{array}{c}
\boldsymbol{\tau} \\
-\boldsymbol{w}
\end{array}\right]=\left[\begin{array}{c}
\boldsymbol{J}_{H}^{T} \\
-\boldsymbol{G}_{H}
\end{array}\right] \boldsymbol{f}+\left[\begin{array}{c}
\boldsymbol{g}_{q} \\
\boldsymbol{o}
\end{array}\right]
$$

where $\boldsymbol{g}_{q}$ denotes the gravitational term of robot and $\boldsymbol{g}_{q}=$ $\partial \sum_{i, j} U_{i j}^{T} / \partial \boldsymbol{q}$ where $U_{i j}$ is the potential energy of the $j$ th link of the $i$ th finger due to the gravity force.

Next, we consider frictional constraints. At $C_{i j}$ which is F-point, it can be written by

$$
\mathcal{F}_{f i j}=\left\{\boldsymbol{f}_{i j}|| \boldsymbol{T}_{i j} \boldsymbol{f}_{i j} \mid \leq \mu_{i j} n_{f_{i j}}, n_{f_{i j}} \geq 0\right\} .
$$

At $C_{i j}$ which is N-point, it can be written by

$$
\mathcal{F}_{n i j}=\left\{n_{f_{i j}} \mid n_{f_{i j}} \geq 0\right\} .
$$

Aggregating for all contact points, we obtain

$$
\begin{array}{r}
\mathcal{F}=\left\{\boldsymbol{f} \mid \boldsymbol{f}_{i j} \in \mathcal{F}_{f i j},{ }^{\forall} C_{i j}\right. \text { which is F-point, } \\
\\
\left.n_{f_{i j}} \in \mathcal{F}_{n i j},{ }^{\forall} C_{i j} \text { which is N-point }\right\} .
\end{array}
$$

\section{New manipulability measures}

Here, we present new manipulability measures based on TVS. In order to reduce the computational effort, we take 3 steps to derive the measures. First, we derive minimum joint torques required to balance any external wrench contained in REWS. Next, from the derived minimum required joint torques and TVS, we derive usable maximum joint velocities. Lastly, we derive generable object velocity from the usable maximum joint velocities.

We approximate the frictional constraint (10) with a $n_{\text {fric }^{-}}$ side convex polyhedral cone circumscribed in the friction cone [10]. Then, (12) becomes

$$
\mathcal{F}_{\text {lin }}=\{\boldsymbol{f} \mid \boldsymbol{V} \boldsymbol{f} \leq \boldsymbol{o}\}
$$

where $\boldsymbol{V} \in \mathcal{R}^{L_{v} \times L_{c}}$.

Step 1: We derive minimum required joint torque, $\left|\tau_{i j_{m r}}\right|$, to balance any external wrench contained in REWS. First, we consider the case when REWS is given as a convex polyhedron $\mathcal{S}_{r e w}^{\text {pol }}$. From (4), (9), (13), we can get $\left|\tau_{i j_{m r}}\right|$ by solving the following linear programming problem.

$$
\begin{gathered}
\underset{\left|\tau_{i j_{m r}}\right|, \boldsymbol{f}_{v_{\iota}}}{\operatorname{argmin}} \quad \Sigma_{i, j}\left|\tau_{i j_{m r}}\right| \\
\text { subject to }\left|\tau_{i j_{m r}}\right| \leq\left|\tau_{i j_{m a x}}\right|\left(j=1, \cdots, M_{i}, i=1, \cdots, N\right) \\
-\boldsymbol{\tau}_{m r} \leq \boldsymbol{J}_{H}^{T} \boldsymbol{f}_{v_{\iota}}+\boldsymbol{g}_{q} \leq \boldsymbol{\tau}_{m r} \\
\boldsymbol{V} \boldsymbol{f}_{v_{\iota}} \leq \boldsymbol{o}, \boldsymbol{G}_{H} \boldsymbol{f}_{v_{\iota}}=-\boldsymbol{w}_{v_{\iota}}\left(\iota=1, \cdots, n_{\text {rew }}\right)
\end{gathered}
$$

where $\boldsymbol{\tau}_{m r}=\operatorname{col}\left[\left|\tau_{i j_{m r}}\right|\right]$.

Next, we consider the case when REWS is given as a ellipsoid $\mathcal{S}_{\text {rew }}^{\text {elip }}$. From (9) and (13), The conditions to balance 
a certain external wrench $\hat{\boldsymbol{w}}_{e x}=\boldsymbol{M}_{w} \boldsymbol{w}_{e x}$ can be written by

$$
\begin{aligned}
& \boldsymbol{A}_{w 1} \hat{\boldsymbol{w}}_{e x}+\boldsymbol{A}_{w 2} \boldsymbol{k}_{w}+\boldsymbol{A}_{w 3} \boldsymbol{\tau}_{m r} \leq \boldsymbol{b}_{w}, \\
& \boldsymbol{A}_{w 1}=\left[\begin{array}{c}
-\boldsymbol{J}_{H}^{T}\left(\boldsymbol{M}_{w} \boldsymbol{G}_{H}\right)^{+} \\
\boldsymbol{J}_{H}^{T}\left(\boldsymbol{M}_{w} \boldsymbol{G}_{H}\right)^{+} \\
\left.-\boldsymbol{V}\left(\boldsymbol{M}_{w} \boldsymbol{G}_{H}\right)^{+}\right)
\end{array}\right], \\
& \boldsymbol{A}_{w 2}=\left[\left(\boldsymbol{J}_{H}^{T} \boldsymbol{\Xi}\right)^{T}\left(-\boldsymbol{J}_{H}^{T} \boldsymbol{\Xi}\right)^{T}(\boldsymbol{V} \boldsymbol{\Xi})^{T},\right]^{T} \\
& \boldsymbol{A}_{w 3}=\left[-\boldsymbol{I}^{T}-\boldsymbol{I}^{T} \boldsymbol{O}^{T}\right]^{T}, \boldsymbol{b}_{w}=\left[-\boldsymbol{g}_{q}^{T} \boldsymbol{g}_{q}^{T} \boldsymbol{o}^{T}\right]^{T},
\end{aligned}
$$

where $\left(\boldsymbol{M}_{w} \boldsymbol{G}_{H}\right)^{+}$denotes the psedo-inverse matrix of $\boldsymbol{M}_{w} \boldsymbol{G}_{H}, \boldsymbol{\Xi}$ denotes an orthogonal matrix whose columns form bases of the null space of $\boldsymbol{M}_{w} \boldsymbol{G}_{H}$, and $\boldsymbol{k}_{w}$ denotes an arbitrary vector.

Now, we will consider the set (15) with respect to $\left[\hat{\boldsymbol{w}}_{e x}^{T} \boldsymbol{\tau}_{m r}^{T}\right]^{T}$. We transform the set (15) which is expressed by H-representation [11] (intersection of half spaces) to its V-representation (convex hull of points and directions).

$$
\left\{\boldsymbol{x}_{w}=\Sigma_{i=1}^{n_{A w}} \lambda_{i} \boldsymbol{x}_{w v i}, \sum_{i=1}^{n_{A w}} \lambda_{i}=1, \lambda_{i} \geq 0\right\}
$$

where $\boldsymbol{x}_{w}=\left[\begin{array}{lll}\hat{\boldsymbol{w}}_{e x}^{T} & \boldsymbol{k}_{w}^{T} & \boldsymbol{\tau}_{m r}^{T}\end{array}\right]^{T}, \boldsymbol{x}_{w v i}$ denotes the vertex and $n_{A w}$ denotes the number of the vertices. We project the set onto the space of $\left[\hat{\boldsymbol{w}}_{e x}^{T} \boldsymbol{\tau}_{m r}^{T}\right]^{T}$ by multiplying the following matrix from the left side: $\left[\begin{array}{lll}I & O & O \\ O & O & I\end{array}\right]$. After that, we transform it to its H-representation.

$$
\hat{\boldsymbol{A}}_{w 1} \hat{\boldsymbol{w}}_{e x}+\hat{\boldsymbol{A}}_{w 3} \boldsymbol{\tau}_{m r} \leq \hat{\boldsymbol{b}}_{w}
$$

$\hat{\boldsymbol{A}}_{w 1}, \hat{\boldsymbol{A}}_{w 3}$ and $\boldsymbol{b}_{w}$ are matrices and vector resulted from the transformation. Note that the above two transformations can be done by cdd library [11].

Let $\hat{\boldsymbol{A}}_{w 1}=\operatorname{col}\left[\hat{\boldsymbol{a}}_{w 1_{i}}^{T}\right]$. Here, we regard the set (16) as the set for the $\hat{\boldsymbol{w}}_{e x}$ space. Generally, the distance between the origin and a hyperplan $\boldsymbol{a}^{T} \boldsymbol{x}=b$ in the space of $\boldsymbol{x}$ (where $\boldsymbol{a}$ and $b$ are constants) is given by $b /|\boldsymbol{a}|$. Therefore, to balance any arbitrary $\hat{\boldsymbol{w}}_{e x}$ contained in REFS, namely $\hat{\boldsymbol{w}}_{e x}$ satisfying $\left|\hat{\boldsymbol{w}}_{e x}\right| \leq 1$ (see (5)), $\boldsymbol{\tau}_{m r}$ should hold the following condition.

$$
\operatorname{col}\left[\left|\hat{\boldsymbol{a}}_{w 1_{i}}\right|\right]+\hat{\boldsymbol{A}}_{w 3} \boldsymbol{\tau}_{m r} \leq \hat{\boldsymbol{b}}_{w} .
$$

Hence, we can get minimum required joint torque $\left|\tau_{i j_{m r}}\right|$ by solving the following linear programming problem.

$$
\begin{gathered}
\underset{\substack{\operatorname{argmin} \\
\left|\tau_{i j_{m} r}\right|}}{\operatorname{argmin}}\left|\sum_{i, j}\right| \tau_{i j_{m r}} \mid \\
\text { subject to }\left|\tau_{i j_{m r}}\right| \leq\left|\tau_{i j_{m a x}}\right|\left(j=1, \cdots, M_{i}, i=1, \cdots, N\right) \\
\operatorname{col}\left[\left|\hat{\boldsymbol{a}}_{w 1_{i}}\right|\right]+\hat{\boldsymbol{A}}_{w 3} \boldsymbol{\tau}_{m r} \leq \hat{\boldsymbol{b}}_{w}
\end{gathered}
$$

If we cannot get the solution at (14) or (17), $\alpha_{\max }^{\text {all }}=\alpha_{\max }=$ 0 . Otherwise, we continue the following steps.

Step 2: Using the derived $\left|\tau_{i j_{m r}}\right|$ and (1), we derive maximum usable joint velocity $\left|\dot{q}_{i j_{U \max }}\right|$.

Step 3: The usable joint velocity set for every joint is expressed by

$$
\left\{\dot{q}_{i j}=-\eta_{1}\left|\dot{q}_{i j_{U \max }}\right|+\eta_{2}\left|\dot{q}_{i j_{U \max }}\right|, \Sigma_{k=1}^{2} \eta_{k}=1, \eta_{k} \geq 0\right\}
$$

where $\eta_{k}$ is a parameter to express the convex polyhedron. The usable joint velocity vector set for every finger is given by the direct sum of the usable joint velocity sets for all joints of the finger. It can be expressed by

$$
\left\{\dot{\boldsymbol{q}}_{i}=\Sigma_{j=1}^{2^{M_{i}}} \eta_{j} \dot{\boldsymbol{q}}_{i_{v j}}, \Sigma_{j=1}^{2^{M_{i}}} \eta_{j}=1, \eta_{j} \geq 0\right\}
$$

where $\dot{\boldsymbol{q}}_{i_{v j}}$ denotes the vertex of the set. From this set and (7), the generable contact velocity set is given by

$$
\left\{\dot{\boldsymbol{p}}_{C i}=\Sigma_{j=1}^{2^{M_{i}}} \eta_{j} \boldsymbol{J}_{H i} \dot{\boldsymbol{q}}_{i_{v j}}, \Sigma_{j=1}^{2^{M_{i}}} \eta_{j}=1, \eta_{j} \geq 0\right\} .
$$

We transform this into its V-representation.

$$
\boldsymbol{A}_{p i} \dot{\boldsymbol{p}}_{C i} \leq \boldsymbol{b}_{p i}
$$

where $\boldsymbol{A}_{p i}$ and $\boldsymbol{b}_{p i}$ are the matrix and the vector resulted from this transformation. Aggregating the relationship with respect to all fingers, utilizing (8), we get

$$
\hat{\boldsymbol{A}}_{r} \dot{\hat{\boldsymbol{r}}} \leq \boldsymbol{b}_{r},
$$

where $\dot{\hat{\boldsymbol{r}}}=\boldsymbol{M}_{r} \dot{\boldsymbol{r}}, \hat{\boldsymbol{A}}_{r}=\boldsymbol{A}_{r} \boldsymbol{M}_{r}^{-1}, \boldsymbol{A}_{r}=\operatorname{col}\left[\boldsymbol{A}_{p i} \boldsymbol{G}_{H i}^{T}\right]$, $\boldsymbol{b}_{r}=\operatorname{col}\left[\boldsymbol{b}_{p i}\right]$ and $\boldsymbol{M}_{r}$ is the weight matrix, for example, to take the difference of units into consideration. This set is the convex polyhedron expressing the generable object velocity set. Let $\hat{\boldsymbol{A}}_{r}=\operatorname{col}\left[\hat{\boldsymbol{a}}_{r i}^{T}\right]$ and $\boldsymbol{b}_{r}=\operatorname{col}\left[b_{r i}\right]$. As mentioned the above, the distance between the origin and the $i$ th face of the convex polyhedron is given by $b_{r i} /\left|\hat{\boldsymbol{a}}_{r i}\right|$. Then, $\alpha_{\text {max }}^{\text {all }}$ can be obtained by solving the following problem.

$$
\alpha_{\max }^{\text {all }}=\min _{i} \quad b_{r i} /\left|\hat{\boldsymbol{a}}_{r i}\right| \text {. }
$$

$\alpha_{\max }$ can be obtained by solving the following convex quadratic programming problem.

$$
\alpha_{\text {max }}^{2}=\max _{\dot{\hat{\boldsymbol{r}}}} \dot{\hat{\boldsymbol{r}}}^{T} \dot{\hat{\boldsymbol{r}}} \quad \text { subject to } \quad \hat{\boldsymbol{A}}_{r} \dot{\hat{\boldsymbol{r}}} \leq \boldsymbol{b}_{r}
$$

Note that the $\dot{\hat{\boldsymbol{r}}}$ providing $\alpha_{\max }$ expresses the direction which provide the maximum generable object velocity.

The alternative way to get $\alpha_{\max }$ is as follows. We transform (20) to its V-representation.

$$
\left\{\dot{\hat{\boldsymbol{r}}}=\Sigma_{i=1}^{n_{A r}} \eta_{i} \dot{\hat{\boldsymbol{r}}}_{v i}, \Sigma_{i=1}^{n_{A r}} \eta_{i}=1, \eta_{i} \geq 0\right\}
$$

where $\dot{\hat{\boldsymbol{r}}}_{v i}$ denotes the vertex and $n_{A r}$ denotes the number of the vertices. Then, we can get $\alpha_{\max }$ by

$$
\alpha_{\max }^{\text {all }}=\max _{i}\left|\dot{\hat{\boldsymbol{r}}}_{v i}\right| .
$$

If we know the (23), we can also get the volume of generable object velocity set by, for example, qhull [12].

\section{NUMERICAL EXAMPLES}

In order to verify our approach, we show some numerical examples. Fig.3 shows the target system. $\Sigma_{R}$ was placed at the contact point between the object and the base in the configuration shown in Fig.3. $\Sigma_{O}$ was placed at geometric center of the object. The object was a ball with radius of $0.1[\mathrm{~m}]$. The robotic hand was composed of 4 fingers which had all the same structures and have 4 joints. The length of every link was set to $0.1[\mathrm{~m}]$. For the simplicity, we ignored the mass of the link. The actuators attached to all joints were all the same. A gear with reduction ratio of $1 / 23$ was attached on every actuator. Supposing all actuators had the 
same TVS, we used the TVSs expressed by the rigid and dot lines in Fig.1. The base positions of fingers were set to $\left[\begin{array}{llll}-0.1 & -0.05 & 0\end{array}\right]^{T},\left[\begin{array}{lll}0.05 & 0.1 & 0\end{array}\right]^{T},\left[\begin{array}{lll}0.05 & 0 & 0\end{array}\right]^{T}$ and $\left[\begin{array}{lll}0.05 & -\end{array}\right.$ $0.10]$. We set $n_{\text {fric }}=8$ and $\mu_{i j}=0.3$ for all F-points.

we considered the case shown in Fig. 4 where the object was moved in positive $z$ direction by the robot hand. The contact points on Fingers 1, 2 and 4 were set to be F-point. The contact point on Finger 3 was set to be N-point.

The contact positions at the fingers at the initial state were set to $\left[\begin{array}{lll}-0.097 & 0 & 0.074\end{array}\right]^{T},\left[\begin{array}{llll}0 & 0.097 & 0.13\end{array}\right]^{T},\left[\begin{array}{llll}0.097 & 0 & 0.074\end{array}\right]^{T}$, $[0-0.0970 .13]^{T}$. We used the following 2 kinds of REWSs.

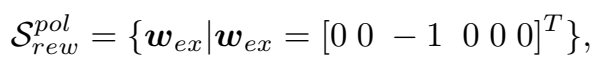

$\mathcal{S}_{\text {rew }}^{\text {elip }}=\left\{\boldsymbol{w}_{\text {ex }} \mid \boldsymbol{w}_{\text {ex }}^{T} \boldsymbol{M}_{w}^{T} \boldsymbol{M}_{w} \boldsymbol{w}_{\text {ex }} \leq 1\right\}, \boldsymbol{M}_{w}=\left[\begin{array}{ll}\boldsymbol{I}_{3 \times 3} & \boldsymbol{O}_{3 \times 3}\end{array}\right]$.

Here, in the setting of $\mathcal{S}_{\text {rew }}^{\text {pol }}$, we supposed that gravitational force with the magnitude of $1[\mathrm{~N}]$ was exerted on the object in $z$ negative direction, while in the setting of $\mathcal{S}_{\text {rew }}^{\text {elip }}$, we supposed that gravitational force with the magnitude of $1[\mathrm{~N}]$ could be exerted on the object in any direction resulted from arm motion. We set $\boldsymbol{M}_{r}$ to normalize $\dot{\boldsymbol{r}}$ as follows:

$$
\boldsymbol{M}_{r}=\left[\begin{array}{cc}
\boldsymbol{I}_{3 \times 3} & \boldsymbol{O}_{3 \times 3} \\
\boldsymbol{O}_{3 \times 3} & 0.1 \boldsymbol{I}_{3 \times 3}
\end{array}\right] .
$$

We computed $\alpha_{\max }^{a l l}$ and $\alpha_{\max }$ when the object moved from the initial state to the final state shown in Fig.4. Fig.5 shows the result. The horizontal axis denotes the $z$ coordinate of $\Sigma_{O}$, and the vertical axis denotes $\alpha_{\max }^{\text {all }}$ or $\alpha_{\max }$. The rigid line expresses the result when using $\mathcal{S}_{\text {rew }}^{\text {elip }}$ and TVS expressed by the rigid line shown in Fig.1, the dashed line expresses the result when using $\mathcal{S}_{\text {rew }}^{\text {pol }}$ and TVS expressed by the rigid line shown in Fig.1, and the dot dashed line expresses the result when using $\mathcal{S}_{\text {rew }}^{\text {elip }}$ and TVS expressed by the dot line shown in Fig.1.

From Fig.5, it can be seen that as the object moved in positive $z$ direction, $\alpha_{\max }^{\text {all }}$ gradually decreased. Around the initial state, every finger (especially Fingers 1 and 3) was in the state where large contact velocity and force in any direction can be generated. We believe it is the reason why $\alpha_{\max }^{\text {all }}$ was large around the initial state. Around the final state, the elbow (third joint) of every finger (especially Fingers 2 and 4) was extended. In this configuration, finger can generate large contact force in the specified direction and cannot in the other directions. It is the same as for contact velocity. This is considered to be the reason why $\alpha_{\max }^{\text {all }}$ was small around the final state. On the other side, $\alpha_{\max }$ gradually increased. Extending the fingers, the generable object velocity in the specified direction became very large.

If comparing the case when using $\mathcal{S}_{\text {rew }}^{\text {pol }}$ with the case when using $\mathcal{S}_{\text {rew }}^{\text {elip }}$, The criteria for $\mathcal{S}_{\text {rew }}^{\text {elip }}$ were smaller. If dealing with external wrenches in all directions, we need larger joint torques for stable grasping. This is one of the reasons.

If comparing the case when using TVS expressed by the rigid line shown in Fig.1 with the case when using TVS expressed by the dot line shown in Fig.1, The criteria for the rigid line were larger. This is considered to be the result that we mainly used the area of TVS where generable joint velocity for the rigid line is larger.

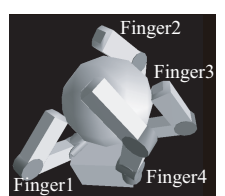

Fig. 3. Target system in numerical examples

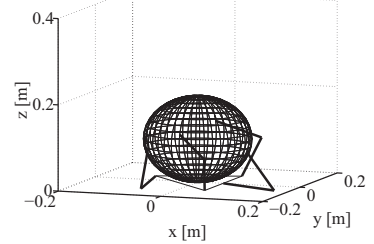

(a)

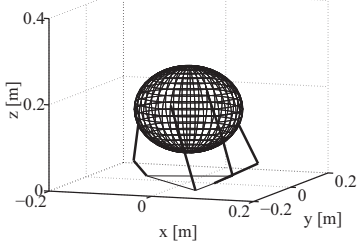

(b)
Fig. 4. (a) initial state, (b) final state

In order to see the effect of friction, we computed when changing the frictional coefficient; $\mu_{i j}=0.01,0.1,0.3$. We used $\mathcal{S}_{\text {rew }}^{\text {elip }}$. Fig.6 shows the result. With the increase of the frictional coefficient, we can get larger $\alpha_{\max }^{\text {all }}$ and $\alpha_{\max }$. When the frictional coefficient increases, the range of applicable contact forces becomes large. Therefore, we can balance external wrench with smaller joint torques, and then generable object velocity became large.

Lastly, we discuss about computational effort. We used linear and quadratic programming problems, which can be solved by polynomial time algorithms, but transformation from $\mathrm{V} / \mathrm{H}$-representation to $\mathrm{H} / \mathrm{V}$-representation cannot be solved by polynomial time algorithms. Therefore, we discuss using the computational time here. In this paper, we used matlab (mathworks) for computation. The CPU of the used PC was Intel Core2 Duo P8800 (@2.66GHz). The CPU time to compute both $\alpha_{\max }^{\text {all }}$ and $\alpha_{\max }$ was from 0.19 to $1.2[\mathrm{~s}]$ (average:0.62[s]) when using $\mathcal{S}_{\text {rew }}^{\text {elip }}$ and TVS expressed by the rigid line in Fig.1, from 0.094 to 0.36 [s] (average:0.18[s]) when using $\mathcal{S}_{\text {rew }}^{\text {pol }}$ and TVS expressed by the rigid line in Fig.1, and from 0.16 to $1.0[\mathrm{~s}$ ] (average:0.62[s]) when using $\mathcal{S}_{\text {rew }}^{\text {elip }}$ and TVS expressed by the dot line in Fig.1.

Note that in our previous method [7], we needed 129[s] of CPU time to compute the simplifier case where the object is grasped by 2 fingers. Therefore, we can say that we could largely reduce the computational efforts.

\section{CONCLUSion}

This paper presented new manipulability measures which can take joint torques required for grasping into consideration. First, we presented a way to derive the minimum required joint torque for stable grasp, using required external wrench set (REWS) given as not only a convex polyhedron but also an ellipsoid. Then, we can take external wrench which can be exerted in any direction into consideration. Next, from the derived minimum required joint torque and the joint torque-velocity pair set which provides the relationship between generable joint torque and velocity, we derived usable maximum joint velocity. We presented a way to deal with a non-convex joint torque-velocity pair set. Lastly, we 


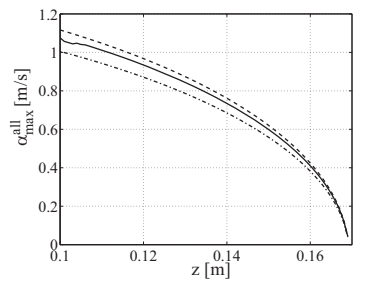

(a) $\alpha_{\max }^{\text {all }}$

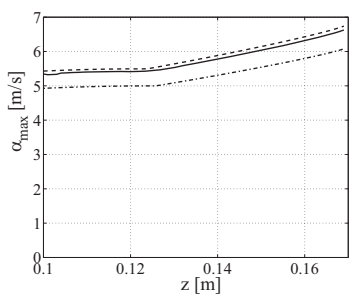

(b) $\alpha_{\max }$
Fig. 5. New manipulability measures $\left(\alpha_{\max }^{a l l}, \alpha_{\max }\right)$. The rigid line expresses the result when using $\mathcal{S}_{\text {rew }}^{\text {elip }}$ and TVS expressed by the rigid line in Fig.1, the dashed line expresses the result when using $\mathcal{S}_{r e w}^{\text {pol }}$ and TVS expressed by the rigid line in Fig.1, and the dot dashed line expresses the result when using $\mathcal{S}_{\text {rew }}^{\text {elip }}$ and TVS expressed by the dot line in Fig.1.

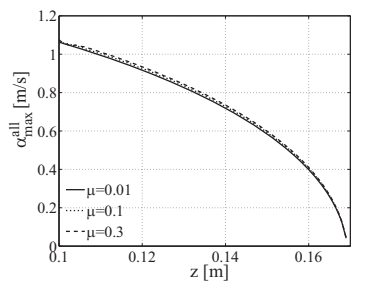

(a) $\alpha_{\max }^{\text {all }}$

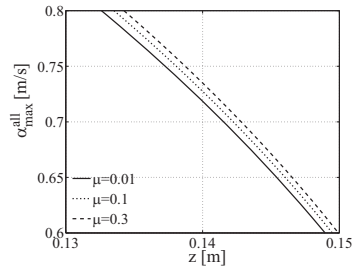

(c) zoomed figure for (a)

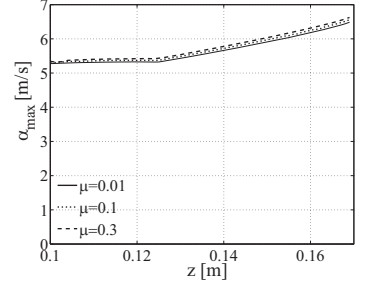

(b) $\alpha_{\max }$

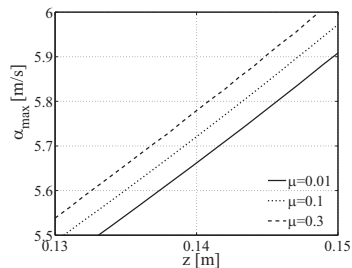

(d) zoomed figure for (b)
Fig. 6. New manipulability measures $\left(\alpha_{\max }^{a l l}, \alpha_{\max }\right)$ when changing the frictional coefficient

derived generable object velocity from the usable maximum joint velocity. The presented way can take the whole space of the generable object velocity into consideration. The validity of our approach was shown by numerical examples, which also showed the proposed measures can include the effect of friction.

\section{APPENDIX}

col A column vector or matrix formed by the following elements. diag A block diagonal matrix.

$N \quad$ Number of fingers.

$M_{i} \quad$ Number of joints of the $i$ th finger $(i=1,2, \cdots, N)$.

$L_{i} \quad$ Number of contact points on the $i$ th finger.

$M \quad$ Number of total joints $\left(=\Sigma_{i=1}^{N} M_{i}\right)$.

$L \quad$ Number of total contact points $\left(=\sum_{i=1}^{N} L_{i}\right)$.

$D \quad 3 / 6$ in $2 / 3$ dimensional space.

$d \quad 2 / 3$ in $2 / 3$ dimensional space.

$\Sigma_{R} \quad$ Reference coordinate frame.

$\Sigma_{O} \quad$ Object coordinate frame fixed at the object.

$\tau_{i j} \quad$ The $j$ th joint torque of the $i$ th finger $\left(j=1, \cdots, M_{i}, i=\right.$ $1, \cdots, N)$.

$q_{i j} \quad$ The $j$ th joint angle of the $i$ th finger $\left(j=1, \cdots, M_{i}, i=\right.$ $1, \cdots, N)$.

$\left|\tau_{i j_{\max }}\right|(\geq 0)$ Generable maximum absolute value of $\tau_{i j}$.

$\left|\dot{q}_{i j_{\max }}\right|(\geq 0)$ Generable maximum absolute value of $\dot{q}_{i j}$.

$\left|\tau_{i j_{U \max }}\right|(\geq 0)$ Usable maximum absolute value of $\tau_{i j}$ determined by currently generating $\dot{q}_{i j}$.

$\left|\dot{q}_{i j_{U \max }}\right|(\geq 0)$ Usable maximum absolute value of $\dot{q}_{i j}$ determined by currently generating $\tau_{i j}$.

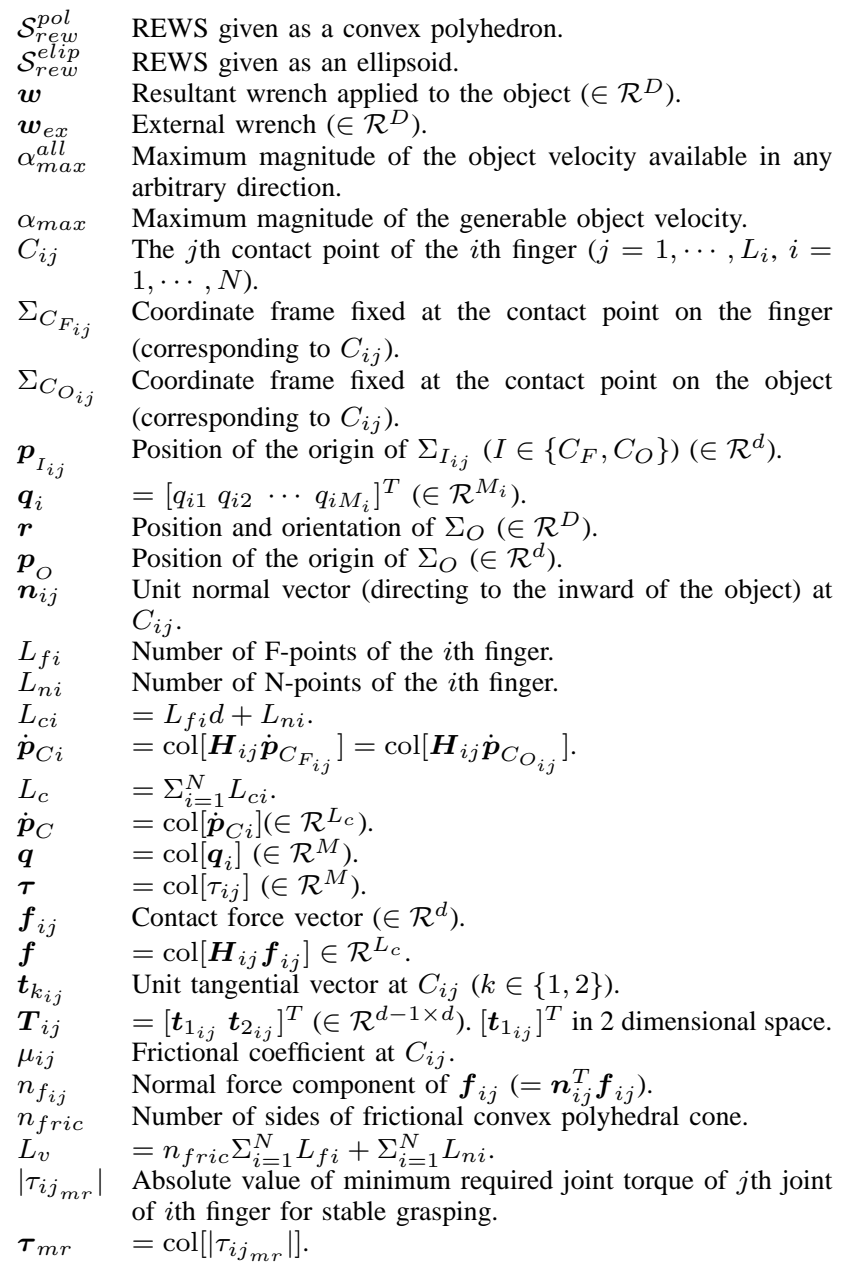

\section{REFERENCES}

[1] T. Yoshikawa, Foundations of Robotics. MIT Press, Cambridge, 1990.

[2] P. Chiacchio et. al., "Global task space manipulability ellipsoids for multiple-arm systems," IEEE Trans. on Robotics and Automation, vol. 7, no. 5, pp. 678-685, 1991, correspondence.

[3] A. Bicchi, C. Melchiorri, and D. Balluchi, "On the mobility and manipulability of general multiple limb robots," IEEE Transactions on Robotics and Automation, vol. 11, no. 2, pp. 215-228, 1995.

[4] A. Bicchi and D. Prattichizzo, "Manipulability of cooperating robots with unactuated joints and closed-chain mechanisms," IEEE Transactions on Robotics and Automation, vol. 16, no. 4, pp. 336-345, 2000.

[5] J. T. Wen and L. S. Wilfinger, "Kinematic manipulability of general constrained rigid multibody systems," IEEE Transactions on Robotics and Automation, vol. 15, no. 3, pp. 558-567, 1999.

[6] F. C. Park and J. W. Kim, "Manipulability and singularity analysis of multiple robot systems: A geometric approach," Proc. of IEEE Int. Conf. on Robotics and Automation, pp. 1032-1037, 1998.

[7] T. Watanabe, "Joint torque-velocity pair based manipulability for grasping system," Proc. of IEEE/RSJ International Conference on Intelligent Robots and Systems, pp. 2264-2270, 2008.

[8] T. Watanabe and T. Yoshikawa, "Grasping optimization using a required external force set," IEEE Transactions on Automation Science and Engineering, vol. 4, no. 1, pp. 52-66, 2007.

[9] T. Watanabe et. al., "Task based hybrid closure grasping optimization for autonomous robot hand," Design and Control of Intelligent Robotic Systems (Springer Berlin / Heidelberg), pp. 425-451, 2009.

[10] J. Kerr and B. Roth, "Analysis of multifingered hands," The International Journal of Robotics Research, vol. 4, no. 4, pp. 3-17, 1986.

[11] K. Fukuda, $c d d / c d d+$ Reference Manual, 1999, [Online]. Available: http://www.ifor.math.ethz.ch/ fukuda/cdd_home/index.html.

[12] C. B. Barber, D. P. Dobkin, and H. Huhdanpaa, "The quickhull algorithm for convex hulls," ACM Transactions on Mathematical Software, vol. 22, no. 4, pp. 469-483, 1996. 